\title{
Interpolação ou Incorporação? Uma análise da trajetória teórica entre a Nova Economia Institucional e a Economia da Informação*
}

\author{
Interpolation or Incorporation? An analysis of the Theoretical trajectory \\ between the New Institutional Economics and the Information Economics
}

Rafael Barbieri Camatta e Alexandre Ottoni Teatini Salles**

\begin{abstract}
Resumo: Nos anos de 1970 surgiram duas importantes correntes em economia, A Nova Economia Institucional (NEI) e a Economia da Informação (EInf). Analisando os principais autores destas agendas de pesquisa, percebe-se que existem conceitos e fundamentos semelhantes, influências cruzadas e trajetórias de desenvolvimento teórico compatíveis entre elas. Entre os principais conceitos discutidos estão: instituições, incerteza, assimetria de informação, racionalidade limitada, oportunismo, risco moral, seleção adversa e custos de transação. Este artigo propõe a hipótese de que a similitude observada não se assemelha a uma interpolação teórica, mas sim a uma incorporação entre a NEI e a EInf, na qual um dos programas de pesquisa se sobrepõe ao outro.
\end{abstract}

Palavras-chave: Instituições. Oportunismo. Assimetria de Informação. Nova Economia Institucional. Economia da Informação

Abstract: In the 1970s two major economic intellectual traditions emerged, The New Institutional Economy (NEI) and the Information Economics (EInf). Analyzing the main authors of these research agendas, it is noted that there are similar concepts and foundations, cross-influences and compatible theoretical development trajectories between them. Key concepts discussed include institutions, uncertainty, information asymmetry, bounded rationality, opportunism, moral hazard, adverse selection, and transaction costs. This paper proposes the hypothesis that the observed similarity does not resemble a theoretical interpolation, but rather an incorporation between NEI and EInf, in which one of the research programs overlaps the other.

Keywords: Institutions. Opportunism. Information Asymmetries. New Institutional Economics. Information Economics

JEL: B25. D83. D86

\footnotetext{
* Submissão: 14/01/2020 | Aprovação: 20/09/2020 | DOI: 10.5380/re.v42i79.71175

** Respectivamente: (1) Doutorando no Programa de Pós-Graduação em Economia da Universidade Federal do Espírito Santo (UFES) | ORCID: 0000-0003-3781-7658 | E-mail: rbcamatta@gmail.com | (2) Professor do Departamento de Economia e do Programa de Pós-Graduação em Economia da Universidade Federal do Espírito Santo (UFES) | ORCID: 0000-0001-9074-2531 |E-mail: aotsalles@gmail.com | Este artigo foi desenvolvido com o apoio da Fundação de Amparo à Pesquisa e Inovação do Espírito Santo (FAPES).
} 


\section{Introdução}

A partir das pesquisas elaboradas por Oliver Williamson (Nobel de Economia em 2009) e Douglass North (Nobel de Economia em 1993) sobre a importância das instituições para a atividade econômica, emerge, ao fim da década de 1970 uma agenda de pesquisa na Ciência Econômica denominada Nova Economia Institucional (doravante NEI). Nesse mesmo período, George Akerlof (Nobel de Economia em 2001), Kenneth Arrow (Nobel de Economia em 1972) e Joseph Stiglitz (Nobel de Economia em 2001) tornaram-se os principais precursores de uma Escola de Pensamento chamada Economia da Informação (doravante EInf) (Stiglitz, 2000)².

Analisando o trabalho desses e de outros autores que deram continuidade a essas agendas de pesquisa, percebe-se que existem conceitos e fundamentos teóricos semelhantes entre elas, tais como: instituições, incerteza, assimetria de informação, racionalidade limitada, oportunismo, risco moral, seleção adversa e custos de transação. Assim, o primeiro objetivo do artigo é apresentar esses princípios teóricos a fim de analisar tais semelhanças (seções 2 e 3). A partir do exame dessa literatura, procura-se comprovar a hipótese de que a agenda de pesquisa da NEI abrange o escopo de análise da EInf. Dessa forma não haveria uma interpolação entre os temas abordados entre as escolas, mas a incorporação teórica da EInf pela NEI. Tal demonstração é o segundo objetivo do estudo (seção 4).

A fim de cumprir tais objetivos, o trabalho foi dividido em quatro seções, além desta introdução. A segunda e terceira apresentam um breve histórico e alguns fundamentos teóricos centrais da NEI e da EInf, respectivamente. A seção 4 correlaciona os conceitos apresentados de modo a demonstrar a hipótese da incorporação da Einf pela NEI. Por fim, breves considerações finais concluem o artigo.

\footnotetext{
${ }^{2}$ Herscovici (2015) propõe uma ressalva a esta nomenclatura. Ele denomina as ideias propostas por Akerlof, Stiglitz e Grossman de "Nova Economia da Informação" de modo a diferenciá-los dos primeiros modelos desenvolvidos sobre o problema da assimetria de informação na Ciência Econômica. De fato, Stiglitz (2000, p.1459) já havia utilizado o termo "new information economics", ou, no mesmo artigo, "modern information economics" (p. 1443) para tratar das diferenças da sua abordagem em relação à de Stigler. Apesar do autor fazer estas distinções, o uso deste termo mencionado por Herscovici (2015) é utilizado de forma pontual na obra de Stiglitz. Neste artigo, optou-se por utilizar indistintamente o termo "Economia da Informação (EInf)" principalmente porque esta denominação tem sido usada por ele de maneira mais geral, inclusive em seu discurso de Nobel (Stiglitz, 2003, 2004).
} 


\section{Elementos fundamentais da estrutura teórica da Nova Economia Institucional (NEI)}

Na História do Pensamento Econômico, os primeiros debates acadêmicos sobre a importância das instituições na estruturação da atividade econômica remontam ao fim do século XIX a partir dos trabalhos seminais de Thorstein Veblen (1857-1929). Ao longo da primeira metade desse século, autores como John Commons (1862-1945), Wesley Clair Mitchell (1874-1948), Walton H. Hamilton (1881-1958) e John Maurice Clark (1884-1963) dentre outros, amplificaram o escopo da abordagem original proposta por Veblen. O debate acadêmico daí proveniente promoveu o surgimento da Economia Institucional, tal como batizada por Hamilton (1919/2017) - mais tarde chamada por Hodgson (1989), Langlois (1989), Mayhew (1989) e Rutherford (1989) de Institucionalismo Original, ou Velho Institucionalismo. Essa Escola assume uma posição de destaque na academia norte-americana no pós-primeira guerra mundial. Contudo, com a consolidação do método matemático (1910 a 1940) na ciência econômica, o Institucionalismo Original passa a ser classificado como "menos rigoroso" e, portanto, "inferior" ao método de investigação baseado em modelagem matemática (Hodgson, 1998).

A partir dos anos 1970, ressurge dentro do mainstream econômico o interesse em temas relacionados as instituições (Williamson, 1996). Emerge então uma nova corrente batizada de Nova Economia Institucional. Um dos princípios teóricos mais gerais dessa Escola propõe que as transações e seus custos definem as estruturas de mercado do sistema econômico. Os mercados, por sua vez, apresentam falhas geralmente relacionadas à incerteza e à racionalidade limitada, que promove um comportamento do tipo oportunista por parte dos agentes econômicos. Assim, no prefácio do livro que pode ser considerado sua magnum opus, Williamson ressalta que a abordagem teórica elaborada pela NEI pode ser compatível com outras interpretações que se propõem a discutir elementos correlacionados aos conceitos mencionados acima. Assim, nas suas palavras: "transaction cost arguments are often best used in conjunction with, rather than to the exclusion of, other ways of examining the same phenomena. I therefore do not propose that a blinkered approach to economic organization proceed heedless of other alternatives" (1985, p. xii).

Antes de discutir os principais conceitos da NEI, vale a pena trazer à memória, à guisa de contextualização, que a abordagem dessa Escola possui como 
principal antecedente o trabalho pioneiro de Coase (1937). Neste, o conceito de transação equivale a um acordo, entre dois ou mais agentes, que busca evitar (ou solucionar) possíveis litígios. Para o autor, a teoria tradicional da firma à época não explicava essa característica marcante acerca do funcionamento das relações contratuais que emergem da atividade econômica. Por isso, Coase propõe que existem custos para o acesso ao mecanismo de preços de mercado decorrentes da dificuldade de formular contratos e de descobrir quais preços são relevantes para sua formulação. Estes foram denominados "custos de transação". A esse respeito, North (1994) aponta que Coase foi o primeiro a relacionar instituições e custos de transação preservando as bases conceituais da teoria marginalista microeconômica. Essas ideias precursoras de Coase serão apresentadas a seguir sob o ponto de vista de Williamson e North.

\subsection{Incerteza e Instituições}

Um dos elementos fundamentais para estruturar toda a gama de conceitos elaborada pela NEI é o de incerteza. O próprio Williamson (1985) afirma que, sem essa discussão, os problemas econômicos tornar-se-iam triviais. A incerteza na obra do autor é caracterizada como comportamental pois está relacionada à conduta oportunista dos agentes. Para ele, o oportunismo é uma atitude pervasiva e assume diversas formas. Dessa maneira, mesmo em situações em que os contratos são bem especificados, podem acontecer surpresas relacionadas ao não cumprimento das regras estabelecidas. Na teoria dos custos de transação, os fenômenos econômicos são únicos, portanto, há sempre espaço para desvios comportamentais não previstos.

Williamson (1985) e North (2005) declaram que a abordagem sobre incerteza proposta pela NEI remete à concepção de Frank Knight, dado que o autor contrastou esse conceito com o de "risco". Em última instância, enquanto risco é passível de mensuração estatística, incerteza não pode ser quantificada desta forma. Isso significa que, para o autor, não existe a possibilidade de modelagem matemática de um ambiente pautado por esse fenômeno. Dessa forma, Knight caracteriza o ambiente econômico como não-ergódigo. Tal fato o levou a utilizar o conceito de moral hazard em seu mais importante livro publicado em 1965. Nas palavras de Williamson (1985, p. 3; 1996, p. 55): “Knight ... acknowledged the importance of studying 'human nature as we know it' (1965, p. 270) and 
specifically identified 'moral hazard' as an endemic condition with which economic organization must contend" (1965, p. 260).

É importante ressaltar que para a NEI, o conceito de instituição deriva imediatamente da incerteza do ambiente econômico. Numa acepção bastante ampla, instituições são constructos humanos que têm o objetivo fundamental de ampliar a capacidade de previsão dos agentes, ou seja, reduzir a incerteza no processo de decisão dos agentes. Nas palavras de North (1990): “Institutions reduce uncertainty by providing a structure to everyday life" (p. 3); de tal forma que: "the major role of institutions in a society is to reduce uncertainty by establishing a stable (but not necessarily efficient) structure to human interaction" (p. 6); por fim, North associa a definição de instituições às "regras do jogo", ou seja, uma forma de conectar os agentes a parâmetros codificáveis e não codificáveis que contribuem para balizar o processo de tomada de decisão: "If institutions are the rules of the game, organizations and their entrepreneurs are the players" (1994, p. 361).

O debate em torno da definição do termo "instituição" é extenso e não pode ser sumariado no âmbito deste artigo ${ }^{3}$. Na esfera da NEI pode-se mencionar duas definições clássicas. A de North (1994, p. 360) foi elaborada nestes termos:

Institutions are the humanly devised constraints that structure human interaction. They are made up of formal constraints (e.g., rules, laws, constitutions), informal constraints (e.g., norms of behavior, conventions, self-imposed codes of conduct), and their enforcement characteristics. Together they define the incentive structure of societies and specifically economies.

A definição de Williamson associa o conceito de instituição ao de custo de transação. No capítulo final do livro de 1985, o autor assevera que:

I conjecture that the general microanalytic strategy employed here will apply elsewhere. This entails making the transaction the basic unit of analysis, ascertaining the underlying attributes of transactions, and aligning institutions (incentives, controls, governance structures) in a discriminating way (Williamson, 1985, p. 385).

Nesta passagem, as instituições são entendidas como incentivos, controles, e estruturas de governança. Logo em seguida, ele afirma:

\footnotetext{
3 Além do debate acerca do conceito de "instituição" travado no interior da NEI e da EInf, há uma extensa discussão sobre o termo no âmbito do Institucionalismo Original. Para tanto, ver, por exemplo, Crawford e Ostrom (1995); Dequech (2009, 2014, 2017); Neale (1987); Hodgson (2000, 2002, 2006, 2007); e Searle (2005).
} 
Transaction costs economics holds that microeconomic institutions play a crucial, subtle, and relatively neglected role in explaining differential economic performance over time, within and between industries, within and between nation states and sociopolitical systems (Williamson, 1985, p. 408) ${ }^{4}$.

Os trechos acima mencionados deixam claro que a concepção de Williamson sobre tal conceito concentra-se nas instituições de governança intrafirma, e toma o institutional environment (ou seja, as regras do jogo) como dado (Williamson, 1996, p. 4-5). Assim sendo, esta pode ser considerada uma das principais diferenças de sua abordagem em relação a de North.

Neste âmbito, a diminuição das assimetrias de informação pode gerar uma diminuição do nível de incerteza da transação. Essa melhora pode ser obtida através do estudo sistemático de determinado fenômeno, de modo que o estoque de conhecimento acumulado permite a interpretação quantitativa de seus possíveis resultados futuros. Essa construção do estoque de conhecimento (informação) pode ser facilitada, dificultada ou impedida pela estrutura institucional vigente.

\subsection{Racionalidade Limitada}

Outro pilar fundamental na construção do programa de pesquisa da NEI é a racionalidade limitada. Para Williamson (1985, 1996, 2000, 2002), racionalidade limitada significa que os agentes são "intencionalmente racionais", porém só atingem esse objetivo parcialmente devido à incapacidade de processar toda a informação disponível ${ }^{5}$. Para ele, o reconhecimento da racionalidade limitada como substrato do processo de decisão dos agentes é que possibilita o estudo de diversas formas de organizações.

Em conformidade, para North (1990), a aceitação de pressupostos comportamentais tais quais os da racionalidade substantiva impediria que a investigação científica abordasse temas fundamentais ao entendimento do sistema econômico e social. $\mathrm{O}$ autor afirma ainda que enquanto a racionalidade substantiva funciona bem numa análise de mercados competitivos com preços estabelecidos $a$

\footnotetext{
${ }^{4}$ Williamson (1985, p. 44) ainda fundamenta sua interpretação sobre o significado do termo instituições concordando com a visão de Iredell Jenkins da seguinte forma: "He observes that human institutions, including law, inherit their major problems and purposes from the general condition of man and holds that the study of mind and of social process is needed to get at the roots".

${ }^{5}$ Williamson (1985, p. 30; 1996, p. 6; 2002, p. 174; 2005, p. 8) extrai esta definição de racionalidade limitada de Herbert Simon, citando-as ipsis litteris nas referências mencionadas neste rodapé.
} 
priori, esta não seria suficiente para compreender um sistema econômico pautado por incerteza e informações assimétricas (North, 2005). Assim, para a NEI, seria necessário utilizar parâmetros comportamentais que estivessem em confluência com o ambiente de análise não-ergódigo. Sobre esse aspecto, North (2005, p. 5) afirma que: "the uncritical acceptance of the rationality assumption is devastating for most of the major issues confronting social scientists and is a major stumbling block in the path of future progress"

Ainda com relação a atividade cognitiva, North $(1990,1994)$ aponta que o agente "aprende" e forma modelos para lidar com sua racionalidade limitada e com o ambiente pautado pela incerteza. Os modelos mentais formulados estão em constante transformação devido ao fluxo ininterrupto de feedbacks e novas experiências. Os agentes tomam suas decisões de acordo com informações incompletas e imperfeitas, portanto não é possível abdicar de uma teoria cognitiva abrangente numa análise de qualquer fenômeno econômico. A visão de mundo dos agentes é moldada pela herança cultural (do passado) e pelas experiências presentes e futuras. As informações são consideradas como fundamentais à eficiência (raramente alcançada) dos mercados. Com o aumento dos custos de informação, o mercado leva os agentes a adquirirem informações para corrigir seus modelos mentais, porém estas geralmente não são suficientes para tanto. Assim, geralmente os agentes tomam suas decisões sob informações incompletas utilizando modelos incorretos.

Segundo North (1994), a teoria marginalista tradicional foi elaborada para explicar a operação de mercados em economias desenvolvidas. Por isso, os autores dessa corrente não buscaram entender o processo histórico no qual essas nações se desenvolveram. Ao identificar essa lacuna, o autor propõe partir do instrumental microanalítico marginalista porém adicionando o tempo histórico e as instituições na análise ${ }^{7}$. Desta forma, afasta-se da teoria marginalista ao abandonar o pressuposto da racionalidade substantiva, porém frisa a importância de preservar os princípios da escassez e da competição propostas por essa corrente.

\footnotetext{
${ }^{6}$ Vale a pena lembrar que, assim como Williamson, o conceito de racionalidade limitada de North (1990, 2005) deriva diretamente de Simon $(1955,1986)$.

${ }^{7}$ De acordo com North (1994, p. 359): "The analytical framework is a modification of neoclassical theory. What it retains is the fundamental assumption of scarcity and hence competition and the analytical tools of microeconomic theory. What it modifies is the rationality assumption. What it adds is the dimension of time". Para o autor, o tempo é uma das dimensões do aprendizado que, por sua vez, possui as seguintes características: cumulatividade; transmissão através da cultura; e capacidade de modificar as instituições.
} 


\subsection{Transação e Estruturas de Governança}

O objetivo central da construção teórica da NEI é estudar a transação e os custos a ela inerentes ${ }^{8}$. Esse aspecto a diferencia das abordagens convencionais cuja análise de custos se restringe ao âmbito da produção e da distribuição de bens. Assim, "a transaction occurs when a good or service is transferred across a technologically separable interface. One stage of activity terminates and another begins" (Williamson, 1985, p.1). Ao analisar as características inerentes à uma transação econômica, Williamson $(1985,1996,2000,2005)$ percebe que existem diversas maneiras diferentes de "transferir" bens e serviços entre "estágios de atividade". Essas são denominadas formas de governança. Por conseguinte, as firmas (assim como os mercados) podem ser consideradas como estruturas de governança.

Pode-se ilustrar os tipos de governança a partir da definição de duas formas opostas de contratação. De um lado, estaria a organização clássica - no sentido de que todas as negociações são realizadas no âmbito do mercado - geralmente utilizada para artigos pouco específicos. Esta se assemelha aos contratos em concorrência perfeita. De outro, estão as transações realizadas sob o paradigma da organização hierárquica, geralmente para ativos (muito) específicos. Nessa situação, a firma detém a produção de todos os insumos e toda a estrutura de distribuição é interna. Por isso, não precisa recorrer ao mercado e estar sujeita aos diferentes tipos de oportunismos inerentes à contratação de fornecedores e distribuidores. Entre os dois extremos estão infinitas formas de combinações mistas em que a firma recorre tanto ao mercado quanto às estruturas hierárquicas.

A partir das estruturas mencionadas acima, nota-se que a existência da especificidade dos ativos é base para uma das principais críticas de Williamson (1985) à teoria microeconômica tradicional uma vez que os modelos convencionais geralmente utilizam produtos homogêneos como mecanismo simplificador. Assim, quanto maior a especificidade, maior a dificuldade de reimplantação, maiores os prejuízos no caso de uma interrupção prematura dos contratos, portanto maior a dependência entre as partes envolvidas na transação.

\footnotetext{
${ }^{8}$ Não é à toa que a NEI muitas vezes é também denominada de Economia dos Custos de Transação (Transaction Cost Economics - TCE). Porém, vale ressaltar que para Williamson, trata-se de coisas diferentes na medida em que a TCE é um ramo da NEI. Em suas palavras: "Indeed, I think of the New Institutional Economics (NIE), of which transaction cost economics (TCE) is a part, as young people's economics" (Williamson, 1996, p. IX). Para uma análise pormenorizada da TCE, ver Pessali (2006).
} 


\subsection{Oportunismo}

As diversas formas de governança têm como principal objetivo reduzir os danos causados pelo comportamento oportunista dos agentes. Na concepção de Williamson (1985, p.47), oportunismo é definido da seguinte forma: "selfinterest seeking with guile. This includes but is scarcely limited to more blatant forms, such as lying, stealing, and cheating. Opportunism more often involves subtle forms of deceit". O termo é classificado pelo autor em duas variações: exante e ex-post. O primeiro equivale as formas oportunistas de distorcer informações pré-contratação, incluindo a prática de deturpar a elaboração do próprio contrato. O segundo refere-se ao comportamento de não seguir à risca as cláusulas estabelecidas em um contrato já firmado. Esses aspectos serão analisados com mais detalhes na seção 4. Para o autor, a racionalidade limitada e o oportunismo são os dois pressupostos comportamentais basilares da teoria dos custos de transação (Williamson, 1996).

North (2003) apresenta sua concepção de oportunismo a partir da utilização da teoria dos jogos em diferentes estratégias (cooperação e não cooperação) mediante o nível de proximidade entre as partes. Segundo ele, em estágios menos desenvolvidos da sociedade, os agentes têm maior retorno pela cooperação devido à proximidade com os indivíduos com quem estão transacionando. Porém, à medida em que ocorre o desenvolvimento social, passam a existir diversas situações em que a não cooperação torna-se atrativa. Nestas, as trocas são majoritariamente impessoais e necessitam de instituições (com destaque para os sistemas jurídico e político) que sejam capazes de reforçar essa cooperação.

Desta maneira, os agentes cooperam quando se deparam com três situações: (i) Quando o jogo é repetido, ou seja, quando não há prazo definido para finalizar as relações de troca. Nessa situação, os agentes não têm incentivos ao oportunismo pois há a certeza de retaliação na negociação posterior; (ii) Quando há informação completa sobre o comportamento dos outros competidores. Se todos os agentes sabem exatamente o comportamento dos concorrentes, não há espaço para oportunismos; e (iii) Quando há poucos jogadores para trocas em sociedades pouco desenvolvidas. Em situações diferentes das 3 mencionadas acima, a cooperação é dificultada. 


\subsection{Custos de Transação}

Definidos os conceitos de incerteza, instituições, racionalidade limitada, transação, estruturas de governança e oportunismo, é importante examinar as interrelações destes com a concepção de "custos de transação". Estes são semelhantes ao atrito observado na física ${ }^{9}$ e existem, assim como o oportunismo, em dois tipos: (i) custos ex-ante, relativos a redigir, negociar e salvaguardar um acordo; e (ii) os custos ex-post, relativos aos custos de adaptação dos contratos, custos de barganha e custos de criação de vínculos para efetuar compromissos seguros (Williamson, 1985, p. 20-21) ${ }^{10}$.

North $(1991,1994)$ concorda com essa abordagem de Williamson acerca da natureza dos custos de transação afirmando que estes referem-se aos custos de especificar o que está sendo trocado e garantir acordos entre as partes. Segundo North (1990), só seria possível entender o papel e o funcionamento das instituições a partir de dois pilares: uma teoria comportamental (discutida acima) e uma teoria de custos de transação.

Esses custos são determinados pela estrutura institucional e pela tecnologia vigentes. A esse respeito, o autor ressalta que as instituições econômicas e políticas reduzem, além dos custos de transação, os custos de produção determinados pela tecnologia. Para o autor, a análise econômica deveria ser abordada historicamente de modo a desvendar como as instituições, políticas e econômicas, evoluem de modo a criar ambientes que favorecem (ou não) o aumento da produção.

Em suma, num ambiente de incerteza pautado por racionalidade limitada, a atividade econômica está sujeita a comportamento oportunista por parte dos agentes. Esse comportamento implica em custos de planejamento, adaptação, e monitoramento, ou seja, em custos de transação. Por sua vez, esses variam de acordo com a frequência da transação, assim como com o nível de especificidade do produto ou serviço transacionado. De acordo com essas variações, as firmas decidem por estruturas de governança diferenciadas. As instituições funcionam como as regras do jogo do sistema cujo objetivo primordial é reduzir as incertezas

\footnotetext{
${ }^{9}$ Esta analogia foi elaborada por Williamson. Em suas palavras (1985, p. 19): "Transaction costs are the economic equivalent of friction in physical systems. The manifold successes of physics in ascertaining the attributes of complex systems by assuming the absence of friction scarcely require recounting here. Such a strategy has had obvious appeal to the social sciences. Unsurprisingly, the absence of friction in physical systems is cited to illustrate the analytic power associated with 'unrealistic' assumptions".

${ }^{10}$ Segundo Williamson (1985, p. 18; 1996, p. 5) "Kenneth Arrow has defined transaction costs as the 'costs of running the economic system". A essa definição, Williamson adiciona as dimensões temporais apontadas.
} 
inerentes ao sistema, economizar custos de transação, gerando assim maior eficiência econômica.

Após examinar brevemente os conceitos fundamentais da NEI formulados por seus mais eminentes disseminadores, a seção seguinte apresentará a base teórica fundamental da Economia da Informação (EInf).

\section{Economia da Informação (EInf)}

A denominação "Economia da Informação" (EInf) seguirá ipsis litteris a definição empregada por Joseph Stiglitz (seu principal expoente contemporâneo) em seu artigo seminal "The Contributions of The Economics of Information to Twentieth Century Economics" (2000). Essa subdisciplina da ciência econômica trata primordialmente de problemas de informação imperfeita, dos custos de obtenção dessa informação, e de como esta gera diferentes comportamentos nos agentes econômicos. Segundo Stiglitz (2000), diversos autores do século XIX como Adam Smith (1723-1790), Alfred Marshall (1842-1924), Max Weber (18641920), Jean Charles Sismondi (1773-1842) e John Stuart Mill (1806-1873) já estavam cientes da importância dos problemas referentes à obtenção de informação no processo de tomada de decisão. Porém, coube aos autores da EInf - na segunda metade do século XX - a sistematização e modelagem dessas questões. Nas palavras de Stiglitz (2000, p. 1446): "Until the development of information economics, models in which economic systems had to adjust to new information were not formulated".

Nesse período do século XX, alguns autores da escola de Chicago abordaram a problemática informacional utilizando o paradigma marginalista de maximização da utilidade, tal como estabelecido nos manuais de microeconomia convencionais. O caso de maior destaque é de Stigler (1961). Seu modelo descreve o equilíbrio quando há igualdade entre o custo marginal de obter a informação e o benefício marginal de obtê-la. Em outras palavras, o autor trata as informações como se fossem commodities. As imperfeições de mercado poderiam ser explicadas pela presença de "custos de transação". Esses, por sua vez, detêm um significado completamente diferente do apresentado na seção anterior. Na interpretação de Stigler, estes seriam custos para adequar as imperfeições dos mercados. Em última instância, ao adicionar os custos de transação aos custos de 
produção, a análise poderia ser desenvolvida a partir de um modelo sem falhas de mercado (veja a este respeito Stiglitz, 2000).

Sob a perspectiva da História do Pensamento Econômico, a EInf surge como uma crítica aos modelos de equilíbrio geral iniciados por Arrow-Debreau na década de 1950. Para Stiglitz (2000, 2009), esses modelos tratam a questão informacional de forma secundária pois o sistema de preços é capaz de informar perfeitamente a todos os agentes envolvidos na transação. Desta forma, o preço pago por cada bem equivale a sua qualidade e não há "desvio" de comportamento envolvido na negociação entre bens e serviços. Essa suposição abstrai o problema informacional de modo a concentrar a análise na alocação dos recursos entre vários mercados (principal objetivo desses modelos).

Em contraponto, na EInf o sistema de preços é incapaz de suprir sequer as informações a respeito da escassez dos bens transacionados, ou seja, mesmo em mercados mais simples, os preços não seriam definidos pela relação entre oferta e demanda. Assim, surgem problemas de assimetrias de informação sob a forma de falhas de mercado. Alguns dos modelos mais disseminados tratam da relação entre empregador e empregado, de segurador e segurado, e entre o emprestador e o tomador de empréstimo.

Furubotn e Ritcher (2005) incluem nos problemas de agência toda a literatura de política monetária acerca do dilema regras versus discricionariedade. Neste, a autoridade monetária seria capaz de agir oportunisticamente através de quebras de regras definidas. Desta maneira, como os ganhos de política monetária expansionista se restringem ao curto prazo, o resultado de longo prazo equivale sempre a um aumento inflacionário, ou seja, em perdas de natureza financeira para o principal (sociedade como um todo). Em suma, esses modelos geralmente discorrem sobre a contraposição de interesses entre as partes utilizando a terminologia agente-principal, em que o primeiro é responsável pela ação e tenta obter vantagens sobre o segundo devido à sua falta de informação e ao custo incorrido na obtenção desta.

Na EInf, a informação não pode ser tratada como um bem comum tal qual em Stigler (1961), pelas seguintes razões: (i) esta é não rival e geralmente não exclusiva, tal qual bens públicos ${ }^{11}$; (ii) cada "peça de informação" é diferente (não

${ }^{11}$ Desta forma, para Stiglitz (2014), informações e conhecimento podem ser classificados como bens públicos tal como propõe a definição de Samuelson (1954). 
homogênea), fato que impossibilita análises agregadas de compra e venda; e (iii) o conteúdo informacional só pode ser revelado ex-post à transação, caso contrário, não há motivo para obtenção de informação já conhecida. Devido a essas características, o mercado informacional pode ser classificado como imperfeito, e qualquer análise nesse âmbito deve levar em conta os problemas de apropriação e reputação envolvidos nas transações (Stiglitz, 2000).

Tendo isso em mente, pode-se elencar as principais contribuições da EInf tal como se segue: a) análise sistematizada de como a informação é criada e como se dissemina; b) ampliação do escopo da ciência econômica para outras dimensões que não a alocação de recursos em ambiente de escassez; c) o sistema de preços não carrega toda a informação necessária aos agentes; e, d) a inclusão de outras formas de transmissão de informação que não os preços.

Dentre a vasta literatura produzida por esta Escola, faz-se necessário destacar dois artigos seminais dos quais derivam as discussões subsequentes, quais sejam, Arrow (1971) e Akerlof (1970). O primeiro compreende a problemática acerca do monitoramento e incentivos no pós-contrato, ou seja, problemas de informação ex-post denominados de risco moral. No segundo, são discutidos problemas de informação ex-ante, nomeados pelo autor de seleção adversa. Neste, Akerlof (1970) relaciona os conceitos de incerteza e qualidade dos bens no âmbito da troca ao discutir os problemas das assimetrias de informação observados num mercado de carros específico (no caso, de carros usados ou lemon markets). No modelo, somente os vendedores (agentes) sabem a condição mecânica real do carro que está à venda. Os consumidores (principais) dependem exclusivamente dos preços para balizar seu nível de demanda. Porém, conforme o autor, os preços não são capazes de informar ex-ante a real qualidade do bem adquirido.

Desta forma, a EInf relaciona qualidade, preços e informação na análise econômica, abdicando assim da premissa de produtos homogêneos tal como proposto pelos modelos microeconômicos convencionais. Nestes, o formato decrescente da curva de demanda deve-se ao fato de que esta depende exclusivamente do preço de forma que não há qualquer motivo para que um preço mais alto seja preferível a um preço mais baixo. Para Stiglitz (1987, 2009), há uma inversão dessa lógica haja vista os preços serem os principais transmissores da informação acerca da qualidade do bem transacionado, o que significa que preço maior equivale a um bem de maior qualidade. Por exemplo, um salário 
baixo leva o empregador a desconfiar da qualidade da mão de obra. Ao abordar essa problemática, abandona-se tanto a concepção de que a oferta se iguala à demanda (dada a possibilidade de contingenciamento) quanto à lei do preço único uma vez que produtos com mesmas características não possuem necessariamente o mesmo preço.

De acordo com a EInf, as instituições surgem para contrabalancear a influência da incerteza acerca da qualidade do produto. Algumas destas são: garantias, marcas, franquias e certificações (diplomas, licenças, cadeias de estabelecimentos). Essas instituições diminuem a incerteza acerca da qualidade do que é produzido de modo a estimular as relações de troca (Akerlof, 1970). Da mesma forma, Stiglitz (2009) aponta que um arranjo institucional adequado pode reduzir o comportamento free rider de modo a responsabilizar o agente pelos danos impostos ao principal.

Na passagem abaixo, Stiglitz (2000, p. 1459) deixa claro a postura da EInf com relação à importância das instituições na teoria econômica:

In standard neoclassical economics the deep properties of an
economy-preferences and technology-determine outcomes. Not just
distribution, but history and institutions did not matter. But there are
natural irreversibilities associated with the creation of knowledge:
history has to matter. Indeed, economies with the same deep
properties could have markedly different equilibria. The new
information economics not only showed that institutions mattered,
and helped explain why the institutions arose and the forms they took,
but showed why they mattered. At the same time, it dispelled a
growing misconception (...) that nonmarket institutions arose to
address market failures, and that in doing so, they restored the
efficiency of the economy.

Desta forma, para a EInf as instituições importam. Elas importam tanto que não é suficiente somente entendê-las em seu estado presente. A compreensão da criação e distribuição da informação só é possível a partir da investigação dos fenômenos históricos (irreversíveis) responsáveis pela formação e modificação dessas instituições.

Em suma, esta seção apresentou alguns pontos fundamentais apresentados por Arrow, Akerlof e Stiglitz na construção da EInf. Tendo isso em mente, a próxima seção aponta as principais confluências (e divergências) teóricas entre essa Escola e a NEI. 


\section{Confluências e divergências}

Conforme apresentado nas seções anteriores, para a NEI e a EInf, o ambiente econômico é pautado primordialmente pela incerteza e racionalidade limitada, que podem ser representadas pela presença ubíqua de informações incompletas na atividade econômica. Esta tríade (incerteza - racionalidade limitada - informações incompletas) baliza as construções teóricas formuladas por ambas as Escolas. A partir desses três aspectos surgem comportamentos oportunistas e problemas informação. Segundo Hercovici (2015), ambas surgem dentro da abordagem neoclássica e progressivamente passam a apresentar incongruências com a ortodoxia, gerando assim novos ramos de pesquisa. De acordo com o autor, é justamente pela adoção dessas três premissas que essas Escolas se afastam do paradigma ortodoxo ${ }^{12}$.

Esse caminho em comum demonstra forte similaridade $\mathrm{e}$ complementariedade de influências. Por exemplo, Arrow é uma das principais influências intelectuais de Williamson. Williamson (1985) ressalta o pioneirismo da proposta de Arrow (1969) que define os custos de transação como o custo de funcionamento do sistema econômico. Além disso, o autor menciona Arrow quando discute a dificuldade dos marginalistas em incorporar a racionalidade limitada, outro ponto fundamental tanto na NEI quanto na EInf.

Na obra de Arrow, o conceito de conhecimento limitado está intimamente correlacionado ao de incerteza, base essencial para o entendimento da economia dos contratos e da informação. Este também aborda as falhas de mercado envolvidas no processo de alocação de recursos como as indivisibilidades e a atribuição de direitos de propriedade. Além desses pontos de convergência, pode-se afirmar que, em seus estudos sobre falhas de informação, a influência de Arrow se estende ao longo de toda a teoria dos custos de transação elaborada por Williamson.

Da mesma forma, ao discutir como as instituições importam para a EInf, Stiglitz (2000, p.1459) afirma: "In a sense, Coase's [1937] work explaining the 'firm' as an institution on the basis of transactions costs can be thought of as a precursor of this work". Portanto, nota-se uma correlação sobre a visão acerca dos custos de transação entre os principais expoentes e fundadores de ambas as Escolas.

\footnotetext{
${ }^{12}$ Como visto anteriormente, apesar do abandono de pressupostos básicos da teoria neoclássica, no caso da NEI, outros pilares foram mantidos, tais como o princípio da escassez e da competição. De fato, Arrow e Williamson admitem que o nível de influência adquirido pela Escola se deu em grande medida pela aceitação destes princípios (Ménard; Shirley, 2008).
} 
Stiglitz afirma ainda que o próprio Coase não concordava com o que veio a ficar conhecido na literatura microeconômica como o "Teorema de Coase". Sobre isso, Stiglitz (2000, p. 1458) argumenta da seguinte forma:

It should be noted that Coase himself did not believe in this formulation commonly attributed to him. There were other grounds for rejecting the hypotheses that efficiency obtained, as long as property rights were well assigned, and that distributional concerns were irrelevant, at least for efficiency, and possibly even for the determination of the nature of outcomes themselves.

Na conceituação de problemas de informação e oportunismo, transparece também uma correlação entre a NEI e a EInf. Assim, segundo Williamson (1985, p. 47):

\begin{abstract}
Ex ante and ex post opportunism are recognized in the insurance literature under the headings of adverse selection and moral hazard, respectively... More generally, opportunism refers to the incomplete or distorted disclosure of information, especially to calculated efforts to mislead, distort, disguise, obfuscate, or otherwise confuse.
\end{abstract}

Desta maneira, a seleção adversa equivale a situações em que somente parte dos agentes possui a informação antes da realização da transação, assim como o oportunismo ex-ante. De forma análoga, o risco moral equivale a situações em que acontecem desvios por parte dos agentes após o estabelecimento dos contratos ocasionando custos para a outra parte, da mesma forma que ocorre numa situação de oportunismo ex-post.

Williamson (1985) declara abertamente que escolheu a utilização do termo oportunismo em detrimento de "risco moral" justamente por esse último se diferenciar da "seleção adversa". O autor optou pela elaboração de um conceito geral que englobasse qualquer tipo de comportamento em que há "self-interest seeking with guile". Em outras palavras, ele procurava um conceito que abrangesse além dos problemas de informação clássicos da EInf, outros ainda não sistematizados. Desta forma, embora o termo "técnico" risco moral possa ser estendido para além de suas primeiras utilizações nos modelos de seguros, "it does not ordinarily elicit sensitivity to the full set of ex ante and ex post efforts to lie, cheat, steal, mislead, disguise, obfuscate, feign, distort, and confuse" (Williamson, 1985, p. 51).

Ao estender essa análise ao conceito de transação, pode-se afirmar que problemas de informação, tal como teorizado na EInf, nada mais são do que 
comportamentos oportunistas específicos que geram os custos de transação na concepção da NEI. Como contrapartida, os agentes estabelecem estruturas de governança e redes institucionais a fim de evitá-los. Entre essas instituições (ou regras do jogo) estão as medidas de enforcement das quais se destacam o monitoramento contratual e os incentivos voltados a reduzir problemas de risco moral/oportunismo ex-post. Assim como a utilização de diplomas e certificações (acompanhados das respectivas instituições responsáveis) para evitar problemas de seleção adversa/oportunismo ex-ante.

Em ambas as correntes, o objetivo dessas instituições é reduzir a incerteza, as falhas de contrato, os custos de transação e a assimetria de informação. Porém, tanto para North (1994) quanto para Stiglitz (2000), nada garante que elas surjam e sejam bem-sucedidas nesse aspecto uma vez que existem instituições capazes de criar e intensificar problemas de informação e as falhas de mercado.

Por sua vez, a premissa da não homogeneidade do produto é adotada sob pontos de vista distintos em ambas as Escolas, porém com o mesmo significado. A EInf defende que não se pode observar perfeitamente a qualidade de produtos e serviços (leia-se, a diferença entre eles), enquanto na NEI a não homogeneidade do produto tangencia a discussão de como as firmas se organizam de formas distintas, dada a especificidade dos bens transacionados.

Quanto à abordagem acerca da governança, há forte semelhança entre as correntes. Para a NEI, a governança equivale à forma de contratação que pode variar entre os dois extremos: contratação clássica (via mercado) e contratação hierárquica (intrafirma). Cada qual com seus custos de transação específicos. O mecanismo que define a forma de contratação está relacionado à especificidade dos ativos e à incerteza inerente às transações. Sobre o ponto de vista do problema agente-principal, as estruturas de governança podem ser entendidas como formas de organização cujo objetivo é reduzir os custos inerentes às assimetrias de informação entre as partes dentro do processo produtivo. Desta forma, uma maior hierarquização deveria ser adotada quando recorrer ao mercado implica em maior incidência de risco moral e seleção adversa. Importante ressaltar que os problemas não cessam nesse tipo de contratação uma vez que existem diversos modelos voltados às assimetrias interiores a firma.

Além da análise efetuada até o momento, é interessante observar como a relação entre a NEI e a EInf aparece em obras recentes dedicadas ao estudo dessas 
Escolas. Em seu manual "Institutions \& Economic Theory: The Contribution of the New Institutional Economics", Furubotn e Ritcher (2005, p. XIV) afirmam ${ }^{13}$ :

The present book views the New Institutional Economics as an amalgam of transaction-cost economics, property-rights analysis, and contract theory, and it is on this interpretation of the field that the book will focus. Our objective is to introduce, and assess critically, the major theoretical contributions that have shaped thinking in the NIE since the field's early beginnings in the 1960s.

Nesta obra, os autores tratam os modelos de agente-principal como uma corrente correlata à NEI, tal como proposto no presente artigo. No capítulo 5, esses modelos de problemas informacionais são apresentados como subdivisão da NEI sobre a alcunha de Teoria do Contrato. Neste capítulo, Furubotn e Ritcher afirmam que os modelos da Teoria do Contrato representam uma tentativa formal de tratar os problemas da NEI, e que, dada a ausência de uma alternativa formal de racionalidade limitada, os modelos recorrem à formulação baseadas na racionalidade perfeita, tal como feito pelos modelos microeconômicos tradicionais. Da mesma forma, a incerteza incalculável é tratada como risco estatístico. Outrossim, tratam os problemas de informação assimétrica entre agente e principal da mesma forma como na primeira onda da EInf representada por Stigler (conforme discutido na seção anterior).

No Handbook of New Institutional Economics de Ménard e Shirley (2008), a literatura agente-principal também é tratada indistintamente como como parte integrante da NEI. Neste livro, Miller (2008) discorre sobre os modelos a partir da Coasean "contingency" theory. Segundo ele, dependendo da tecnologia, grau de assimetria de informação e nível de preferências em relação ao risco, existem diferentes soluções para o problema agente-principal. Em seu panorama, o autor utiliza artigos que tratam de problemas específicos e não referencia os trabalhos clássicos aqui apresentados. Da mesma forma, devido a não preocupação em apresentar a EInf como subdisciplina, Stiglitz figura apenas em uma das referências.

Já no livro New Institutional Economics, a Guidebook, de Brousseau e Glachant (2008), a EInf aparece brevemente sob a denominação de "incentive theory". Segundo os autores, apesar desses trabalhos terem gerado importantes insights com relação a incentivos e identificação de ineficiências econômicas, não

\footnotetext{
${ }^{13}$ Publicado originalmente em alemão em 1996.
} 
abordaram explicações endógenas sobre a existência de instituições extramercado voltadas ao processo coordenação tal como feito pela NEI.

Ainda com relação a obras voltadas a cumprir a função de "manuais de NEI", nota-se que os problemas de informação aqui atribuídos a EInf não são sequer referenciados no livro Essays on New Institutional Economics (Richter, 2015). Neste, a NEI é dividida entre duas vertentes. A primeira é relativa aos autores e ideias aqui apresentadas na seção 2 . A segunda é representada por autores que tratam as instituições como autorreguláveis, e desta maneira não há a utilização do conceito de custos de transação. Nas palavras do autor: "We call it the 'invisible-hand'approach to institutional economics" (Richter, 2015, p. 2).

Em suma, como proposto na introdução, o objetivo deste artigo é estabelecer um paralelo entre os principais fundamentos teóricos da NEI e da EInf. Para tanto, foi necessário lidar com diversos conceitos similares conforme sintetizado no quadro abaixo.

\section{Quadro 1 - Conceitos chave para a NEI e EInf}

\begin{tabular}{|c|c|c|}
\hline CONCEITO & NEI & Einf \\
\hline Incerteza & Não redutível a risco & Não redutível a Risco \\
\hline Instituições & $\begin{array}{c}\text { Redutoras de Custos de } \\
\text { Transação e de Incerteza }\end{array}$ & $\begin{array}{c}\text { Redutoras de problemas de } \\
\text { informação e de Incerteza }\end{array}$ \\
\hline Informação & Assimétrica & Assimétrica \\
\hline Problemas de agência & Ex-Ante/Ex-Post & Seleção Adversa/ Risco Moral \\
\hline Racionalidade & Limitada & Limitada \\
\hline Governança & Formas de Contratação & Agente/Principal \\
\hline Tipo de Bem & Não-Homogêneo & Não-Homogêneo \\
\hline
\end{tabular}

Fonte: Elaboração própria.

De acordo com o quadro acima, pode-se perceber que há completa convergência entre três pilares conceituais que formam a estrutura teórica básica do pensamento de ambas as Escolas, quais sejam, incerteza não redutível a risco, informação assimétrica e racionalidade limitada. Por sua vez, a NEI trata os problemas de agência como oportunismo ex-ante e ex-post enquanto na EInf estes mesmos são denominados seleção adversa e risco moral. Em ambas, as instituições servem como formas de reduzir a incerteza e as assimetrias de informação. Ademais, pode-se mencionar que o conceito de governança é empregado de forma 
diferenciada. Para a NEI a governança está relacionada a formas de contratação, enquanto a EInf trata deste assunto como um problema agente/principal.

Por fim, à luz do debate elaborado acima, pode-se comprovar a hipótese apresentada na introdução deste artigo de que a agenda de pesquisa da EInf foi incorporada no escopo de análise NEI. Isso se deve ao fato de que essa última estende seu objeto de pesquisa para além dos problemas do tipo agente/principal. Isso pode ser claramente observado na comparação da definição de oportunismo de Williamson com os conceitos de risco moral e seleção adversa de Akerlof e Stiglitz. Nota-se também que, devido à obra de North, pode ser creditada à NEI uma discussão do papel das instituições numa perspectiva que ultrapassa a problemática acerca dos conflitos entre e intra firmas.

\section{Considerações finais}

O objetivo deste artigo foi apresentar os principais fundamentos teóricos elaborados pela NEI e EInf e, a partir daí, destacar a confluência entre as Escolas. Assim, a primeira conclusão a ser mencionada é que ambas as Escolas surgem dentro do paradigma de pesquisa microeconômico marginalista e passaram a apresentar incongruências em relação a este ao longo de seu desenvolvimento.

Em segundo lugar, a pesquisa demonstrou que elas possuem influências cruzadas uma vez que tratam de uma problemática geral correlata na ciência econômica, qual seja: como ocorrem os comportamentos oportunistas dos agentes em um ambiente marcado por incerteza, racionalidade limitada e assimetria de informação. Em ambas, as instituições podem funcionar como redutoras desses problemas, sejam eles ex-ante ou ex-post.

Em terceiro lugar, além de demonstrar que há semelhanças entre os principais conceitos e fundamentos, este artigo buscou esclarecer que embora sejam tratadas como Escolas de Pensamento diferentes, pode-se considerar que a Einf foi incorporada pelo escopo de análise da NEI. Esse ponto fica claro dada a conceituação de oportunismo de Williamson (1985). O autor afirma que o conceito de oportunismo em sua obra engloba tanto os problemas de risco moral e seleção adversa tratados pela Einf como outras formas mais sutis de "seeking self-interest with guile". Adicionalmente, essa incorporação foi notada em obras recentes voltadas à apresentação da NEI, tais como as de Furubotn e Ritcher (2005) e Ménard e Shirley (2008). 


\section{Referências}

AKERLOF, G. The market for "lemons": qualitative uncertainty and the market mechanism. Quarterly Journal of Economics, v. 84, n. 3, p. 488-500, 1970.

ARROW, K. The organization of economic activity: issues pertinent to the choice of market versus nonmarket allocation. IN: The analysis and evaluation of public expenditure: the PPB system. Washington: U.S. Government Printing Office, 1969.

ARROW, K. Essays in the theory of risk-bearing. Chicago: Markham Publishing Co., 1971

BROUSSEAU, É.; GLACHANT, J.-M. New institutional economics, a guidebook. New York: Cambridge University Press, 2008.

COASE, R. The nature of the firm. Economica, v. 4, n. 16, p. 386-405, 1937.

CRAWFORD, S.; OSTROM, E. A grammar of institutions. American Political Science Review, v. 89, n. 3, p. 582-600, 1995.

DEQUECH, D. Institutions, social norms, and decision-theoretic norms. Journal of Economic Behavior and Organization, v. 72, n. 1, p. 70-78, 2009.

DEQUECH, D. The Institutions of Economics: A First Approximation. Journal of Economic Issues, v. 48, n. 2, p. 523-531, 2014.

DEQUECH, D. O conceito de instituições e algumas tipologias. In: SALLES, A.; PESSALI, H.; FERNÁNDES, R. (Orgs.). Economia institucional: fundamentos teóricos e históricos. São Paulo: Editora Unesp, 2017.

FURUBOTN, E.; RITCHER, R. Institutions and economic theory: the contribution of the New Institutional Economics. Michigan: The University of Michigan Press, 2005.

GALA, P. A retórica na economia institucional de Douglass North. Revista de Economia Política, v. 23, n. 2, p. 311-323, 2003.

GROSSMAN, S.; STIGLITZ, J. Information and competitive price system. American Economic Review, v. 66, n. 2, p. 246-253, 1976.

HAMILTON, W. A abordagem institucional para a teoria econômica. In: SALLES, A.; PESSALI, H.; FERNÁNDES, R. (Orgs.). Economia institucional: fundamentos teóricos e históricos. São Paulo: Editora Unesp, 2017. 
HERSCOVICI, A. A economia neoclássica: uma análise lakatosiana: da cheia do mainstream até sua implosão. Revista de Economia Política, v. 35, n. 4, p. 780-798, 2015.

HODGSON, G. Institutional economic theory: the old versus the new. Review of Political Economy, v. 1, n. 3, p. 249-269, 1989.

HODGSON, G. On the evolution of Thorstein Veblen's evolutionary economics. Cambridge Journal of Economics, v. 22, n. 4, p. 415-431, 1998.

HODGSON, G. What is the essence of institutional economics? Journal of Economic Issues, v. 34, n. 2, p. 317-329, 2000.

HODGSON, G. The evolution of institutions: an agenda for future theoretical research, Constitutional Political Economy, v. 13, n. 2, p. 111-127, 2002.

HODGSON, G. What are institutions? Journal of Economic Issues, v. 40, n. 1, p. 1-25, 2006.

HODGSON, G. Institutions and individuals: interaction and evolution, Organization Studies, v. 28, n. 1, p. 95-116, 2007.

KNIGHT, F. Risk, uncertainty and profit. New York: Harper \& Row, 1965.

LANGLOIS, R. What was wrong with the old institutional economics (and what is still wrong with the new)? Review of Political Economy, v. 1, n. 3, p. 270-298, 1989.

MAYHEW, A. Contrasting origins of the two institutionalisms: the social science context. Review of Political Economy, v. 1, n. 3, p. 319-333, 1989.

MÉNARD, C.; SHIRLEY, M. Handbook of New Institutional Economics. Berlin: Springer, 2008.

MILLER, G. Solutions to principal-agent problems in firms. In: MÉNARD, C.; SHIRLEY, M. (Orgs.). Handbook of New Institutional Economics. Berlin: Springer, 2008.

NEALE, W. Institutions. Journal of Economic Issues, v. 21, n.3, p. 1177-1206, 1987.

NORTH, D. Institutions, institutional change and economic performance. New York: Cambridge University Press, 1990. 
NORTH, D. Institutions. Journal of Economic Perspectives, v. 5, n. 1, p. 97-112, 1991.

NORTH, D. Economic performance through time. American Economic Review, v. 84, n. 3, p. 359-368, 1994.

NORTH, D. The role of institutions in economic development. Discussion Paper Series n. 2003.2. Geneva: United Nations Economic Commission for Europe, 2003.

NORTH, D. Understanding the process of economic change. Oxford: Princeton University Press, 2005.

PESSALI, H. The rhetoric of Oliver Williamson's transaction cost economics. Journal of Institutional Economics, v. 2, n. 1, p. 45-65, 2006.

RICHTER, R. Essays on New Institutional Economics. Cham: Springer International Publishing, 2015.

RUTHERFORD, M. What is wrong with the new institutional economics (and what is still wrong with the old)? Review of Political Economy, v. 1, n. 3, p. 299$318,1989$.

SALLES, A.; PESSALI, H.; FERNÁNDES, R. Economia Institucional: fundamentos teóricos e históricos. São Paulo: Unesp, 2017.

SAMUELSON, P. The pure theory of public expenditure. Review of Economics and Statistics v. 36, n. 4, p. 387-389, 1954.

SEARLE, J. What is an institution? Journal of Institutional Economics, v. 1, n. 1, p. 1-22, 2005.

SIMON, H. A Behavioral model of rational choice. Quarterly Journal of Economics, v. 69, n. 1, p. 99-118, 1955.

SIMON, H. Rationality in psychology and economics. The Journal of Business, v. 59, n. 4, p. S209-S224, 1986.

STIGLER, G. The economics of information. Journal of Political Economy, v. 69, n. 3, p. 213-225, 1961.

STIGLITZ, J. The causes and consequences of the dependence of quality on price. Journal of Economic Literature, v. 25, n. 1, p. 1-48, 1987. 
STIGLITZ, J. The contributions of the economics of information to the twentieth century economics. Quarterly Journal of Economics, v. 115, n. 4, p. 1441-1478, 2000.

STIGLITZ, J. Information and the change in the paradigm in economics, Part 1. American Economic Review, v. 47, n. 2, p. 6-26, 2003.

STIGLITZ, J. Information and the change in the paradigm in economics, Part 2. American Economist, v. 48, n. 1, p. 17-49, 2004.

STIGLITZ, J. Selected works of Joseph E. Stiglitz, Volume I: information and economic analysis. New York: Oxford University Press, 2009.

STIGLITZ, J. Creating a learning society: a new approach to growth, development, and social progress. New York: Columbia University Press, 2014.

WILLIANSON, O. The economic institutions of capitalism: firms, markets, relational contracting. New York: The Free Press, 1985.

WILLIANSON, O. The mechanisms of governance. New York: Oxford University Press, 1996.

WILLIANSON, O. The New Institutional Economics: taking stock, looking ahead. Journal of Economic Literature, v. 38, n. 3, p. 595-613, 2000.

WILLIANSON, O. The theory of the firm as governance structure: from choice to contract. Journal of Economic Perspectives, v. 16, n. 3, p. 171-195, 2002.

WILLIANSON, O. The economics of governance. American Economic Review, v. 95, n. 2, p. 1-18, 2005. 